\title{
Development of synthesis method of magnetic nanoparticles modified by oleic acid and chitosan as a candidate for drug delivery agent
}

\author{
Ika O. Wulandari ${ }^{1}$, Hermin Sulistyarti ${ }^{1}$, Anna Safitri ${ }^{1}$, Dionysius J. D. H Santjojo ${ }^{2,3}$, Akhmad Sabarudin $^{1,3^{*}}$ \\ ${ }^{1}$ Department of Chemistry, Faculty of Science, Brawijaya University, Malang, Indonesia. \\ ${ }^{2}$ Department of Physics, Faculty of Science, Brawijaya University, Malang, Indonesia. \\ ${ }^{3}$ Research Center for Advanced System and Material Technology, Brawijaya University, Malang, Indonesia.
}

\begin{tabular}{l}
\hline ARTICLE INFO \\
\hline Received on: 19/11/2018 \\
Accepted on: 09/05/2019 \\
Available online: 01/07/2019 \\
\\
\hline Key words: \\
Magnetic nanoparticles, oleic \\
acid, chitosan, doxorubicin, \\
drug loading.
\end{tabular}

\begin{abstract}
In this study, magnetic nanoparticles (MNPs) coated with a combination of oleic acid and chitosan were synthesized by ex situ and in situ coprecipitation methods. Morphology and particle size, crystal structure and crystallite size, chemical structure, and magnetic saturation were characterized by scanning electron microscope (SEM), X-ray diffraction (XRD), Fourrier transform infrared, and vibrating sample magnetometer (VSM), respectively. SEM images showed that better spherical morphology is obtained by ex situ co-precipitation method. The XRD pattern identified that nanoparticles containing $\mathrm{Fe}_{3} \mathrm{O}_{4}$ and $\gamma-\mathrm{Fe}_{2} \mathrm{O}_{3}$. The particles and crystallite size of the nanoparticles tended to decrease with increasing oleic acid to the optimum composition. Further functionalization through the chitosan addition (crosslinked by Tripolyphosphate/sulfate) is contributed to the hydrophilicity properties of nanoparticles. Through VSM analysis, MNPs-oleic acid-chitosan showed superparamagnetic behavior with magnetic saturation reaching $32.63 \mathrm{emu} / \mathrm{g}$. There was a linear correlation between magnetic saturation and $\mathrm{Fe}_{3} \mathrm{O}_{4}$ content of nanoparticles. Drug loading and drug release were carried out by using Doxorubicin. These nanoparticles showed a high drug loading efficiency with lower chitosan composition. Loading efficiency of Doxorubicin is related to the conjugation with carboxylic groups and hydrophobic sites from oleic acid and MNPs.
\end{abstract}

\section{INTRODUCTION}

Cancer is a serious problem in the health sector. Currently, treatment and therapy techniques for cancer sufferers are generally performed with chemotherapy methods and conventional drug administration. In general, drugs use in cancer therapy are classified into three categories, including cytotoxic agents, biological agents, and hormonal agents. However, later it is discovered that the therapy is generally ineffective and inefficient since cancer drugs do not work specifically on cancer cells. In addition, there are also undesired side effects by patients, including hair loss and other bodily organ disorders (Cho et al., 2008; Snipstad et al., 2014; Voicu et al., 2016).

*Corresponding Author

Akhmad Sabarudin, Department of Chemistry, Faculty of Science,

Brawijaya University, Malang, Indonesia.E-mail: sabarjpn@gmail.com
An effort to overcome the drawback is to use a drug delivery agent. Drug delivery agents are formulated to deliver drug substances in biological systems so that they can arrive at specific target cells. This system regulates the effectiveness and stability of drug distribution in the body through drug release control (De Jong and Borm, 2008; Dumitriu and Dumitriu, 1996; Jain, 2008; Ulbrich and Lamprecht, 2010). There are several characteristics which need to be considered in maintaining the effectiveness and stability of nanoparticles as drug delivery agents. These characteristics include size, morphology, and surface functional effects of nanoparticles. They can be affected by various factors, namely, the composition ratio, temperature, $\mathrm{pH}$, type of solvent, reaction time/time, and the nature of material (Banerjee et al., 2016).

Oleic acid is a kind of surfactant with amphiphilic properties consist of long chain hydrocarbon as hydrophobic group and carboxylic acid group as hydrophilic. Modification mechanism of oleic acid on magnetic nanoparticles (MNPs) surface can be occurred by coordination between carboxylic 
anion of oleic acid with the surface, result in protective monolayer (Bloemen et al., 2012; Pereira da Silva et al., 2016; Tran et al., 2015). Oleic acid can be utilized for biomedical application due to their high biocompatibility and low toxicity. In particular, hydrophobic chain has important role to carry hydrophobic drug (Velusamy et al., 2016). However, oleic acid has been limited to soluble in aqueous solution rather than organic solvent due to their long chain hydrocarbon. Therefore, further modification by adding hydrophilic polymer will necessary to improve their solubility. Chitosan can be used for further modification on the surface of MNPs-oleic acid because of their biocompatibility. According to the previous study, chitosan was also effective to control size and shape of nanoparticle. Chitosan nanoparticles can be accomplished through ionotropic gelation method by adding crosslinker. The addition of crosslinker was also carried out to produce spherical nanoparticles and was able to regulate the rate of drug release in its application as a drug delivery agent (Majeed et al., 2013; Singh and Vasundhara, 2015).

In this study, MNPs-based drug delivery agent was modified with a combination of surfactants and natural polymers. The purpose of modification was intended to reduce the agglomeration process and prevent MNPs oxidation. This study investigates the effect of ex situ and in situ on nanoparticle material synthesis of physical and magnetic characteristics in the produced material. The ex situ method was carried out by process phases, including MNPs nanoparticles manufacturing and followed by adding coating agents and crosslinkers, whereas in the in situ method, oleic acid was added before MNPs formation. After MNPs-OA was formed, then coating with chitosan and crosslinker was carried out. In addition, tests were also carried out on the effect of coating and crosslinking agent concentrations. The synthesis material was then characterized by scanning electron microscopy (SEM), Fourier transform infrared (FTIR), X-ray diffraction (XRD), and electron spin resonance to determine the physical properties and magnetic characteristics of the produced material, respectively. The drug loading test was then carried out on nanoparticle material with doxorubicin as a drug model, as well as drug release testing from nanoparticles.

\section{MATERIALS AND METHODS}

\section{Materials}

Some materials and chemicals used in this work were the low molecular weight chitosan (deacetylation degree 75\%-85\%), sodium tripolyphosphate [TPP, $85 \%(\mathrm{w} / \mathrm{w})]$, iron (II) chloride tetrahydrate iron $\left[\mathrm{FeCl}_{2} \bullet 4 \mathrm{H}_{2} \mathrm{O}, 98 \%(\mathrm{w} / \mathrm{w})\right]$, iron (III) chloride hexahydrate $\left[\mathrm{FeCl}_{3} \cdot 6 \mathrm{H}_{2} \mathrm{O}, 99 \%(\mathrm{w} / \mathrm{w})\right]$, ethanol $[\mathrm{EtOH}, 96 \%$ (v/v)], and Dulbecco's Phosphate Buffer Saline (PBS) pH 7.4. In addition, this study also used ammonia water $\left[\mathrm{NH}_{3}, 25 \%(\mathrm{v} / \mathrm{v})\right]$, oleic acid [OA, 99\% (v/v)], sodium sulfate $\left[\mathrm{Na}_{2} \mathrm{SO}_{4}, 99 \%(\mathrm{w} / \mathrm{w})\right]$, acetic acid glacial $\left[\mathrm{CH}_{3} \mathrm{COOH}, 100 \%(\mathrm{v} / \mathrm{v})\right]$, and Doxorubicin $(\mathrm{DOX}) \mathrm{HCl}$, all of which were obtained from Merck.

\section{Magnetic nanoparticles preparations}

MNPs were prepared by mixing $\mathrm{FeCl}_{2} \cdot 4 \mathrm{H}_{2} \mathrm{O}(0.125 \mathrm{~g})$ and $\mathrm{FeCl}_{3} \cdot 6 \mathrm{H}_{2} \mathrm{O}(0.35 \mathrm{~g})$ salts into $50-\mathrm{ml}$ distilled water, where mole ratio of $\mathrm{Fe}^{2+} / \mathrm{Fe}^{3+}$ was $1 / 2$. Then solution of 3-M ammonia water added to reach $\mathrm{pH}>9.2$ through a syringe pump with a speed of $10 \mathrm{ml} /$ hour while stirred with a magnetic stirrer, then washed with distilled water several times till neutral $\mathrm{pH}$.

\section{Modification of nanoparticle surfaces with oleic acid and chitosan polymers by ex situ method}

Exsitu coprecipitation method was designed to investigate the effect of adding surface modifiers after MNPs formation on the physical properties and magnetic characteristics of nanoparticles. The synthesized MNPs were modified by the addition of oleic acid $(0.5,1$, and $1.5 \mathrm{ml})$ dissolved in ethanol (volume ratio $\mathrm{OA}: \mathrm{EtOH}$ $=1: 4)$ at $80^{\circ} \mathrm{C}$ and stirred with a magnetic stirrer for 30 minutes. The nanoparticles were then washed with ethanol and distilled water several times. A $3 \mathrm{ml}$ of chitosan solution $(0.5 \%$ and $1 \%$ $\mathrm{w} / \mathrm{v}$ ) was then added to MNPs-OA nanoparticles and stirred for 1 hour. The crosslinking agent in the form of $3 \mathrm{ml} \mathrm{TPP}(2.5 \%, 5 \%$, and $7.5 \% \mathrm{w} / \mathrm{v})$ and sulfate $(7.5 \% \mathrm{w} / \mathrm{v})$ combination was also added through a syringe pump with a speed of $10 \mathrm{ml} /$ hour while stirred with the magnetic stirer for 1 hour. The formed colloidal MNPs$\mathrm{OA}$-chitosan was then washed with distilled water to reach $\mathrm{pH} \pm 7$. Nanoparticles were then dried through the freeze-drying method. The dried samples were characterized by SEM, FTIR, XRD, and VSM.

\section{Modification of nanoparticle surfaces with oleic acid and chitosan polymers by in situ method}

As a comparison, in situ coprecipitation method was designed to see the effect of adding surface modifiers in the initial process of MNPs formation to properties of nanoparticles. The addition of oleic acid was carried out during the formation process of MNPs, namely, when Fe salt was conditioned in alkaline atmosphere by adding 3-M water ammonia to reach $5.5 \mathrm{ml}$. Oleic acid was added to $\mathrm{Fe}$ salt solution via a micro pipette $(0.1$ and $1 \mathrm{~mL}$ ). The solution was stirred with a magnetic stirrer and the addition of bases was continued until the $\mathrm{pH}$ of solution reached $>9.2$. Furthermore, the formed nanoparticles were coated again with $1 \%$ chitosan and crosslinker combination of TPP:sulfate [concentration ratio $(7.5 \%: 7.5 \% \mathrm{w} / \mathrm{v})$ ] through a syringe pump with a speed of $10 \mathrm{ml} /$ hour while stirred on the magnetic stirrer. The formed MNPs-OA-chitosan nanoparticles were then washed with distilled water to reach $\mathrm{pH} \pm 7$. Next, the samples were dried with freeze drying method and characterized.

\section{Characterization of magnetic nanoparticles}

The particle size and morphology of nanoparticles magnetic surface with or non-coating oleic acid-chitosan were determined by SEM (SEM SU3500, Hitachi High-Technologies Corporation, Tokyo). Because the particles tend to form clusters, Image $\mathrm{J}$ software was used to assess the particle size of MNPs. Diffractogram patterns of Nanoparticles were analyzed by XRD (Ultima IV, Type II diffractometer, Rigaku Americas Corporation) by using $\mathrm{Cu} \mathrm{K} \alpha$ radiation $(\lambda=1.5406 \AA)$ with a range of $2 \theta$ from $25^{\circ}$ to $65^{\circ}$ (interval $0.02 \theta$ ), $50 \mathrm{kV} / 60 \mathrm{~mA}$, and graphite crystal monochromator. Furthermore, the determination of crystallite size was carried out by processing XRD diffractogram data with modified Debye-Scherrer equation (Monshi et al., 2012). The plot of equation was shown in Figure 1.

The calculation of iron oxide phase abundance and lattice parameters was carried out by Diffraction (MAUD) software 
compared with ICSD collection code 26410 and 172905 data for $\mathrm{Fe}_{3} \mathrm{O}_{4}$ and $\gamma-\mathrm{Fe}_{2} \mathrm{O}_{3}$, respectively. Perkin-Elmer spectrum 400, USA was used to obtain FTIR spectra in the range of 4,000-400 $\mathrm{cm}^{-1}$ by using $\mathrm{KBr}$ (potassium bromide) pellets for nanoparticle samples. FTIR spectra were used to identify the presence of functional groups from MNPs and MNPs-OA-chitosan samples. Magnetic properties of MNPs-OA-chitosan samples which included magnetization saturation and coercivity were measured by VSM (C7-10A, TOEI Industry, Co. Ltd, Tokyo, Japan).

Determination of drug loading efficiency and drug loading capacityin nanoparticle samples

The $10 \mathrm{mg}$ of MNPs-OA-chitosan dried with freeze dryer was dissolved in 30-ml PBS containing DOX for 1 hour while stirred with a magnetic stirrer. The supernatant was taken for further analysis with a Visible spectrophotometer (UV-Vis 1601, Shimadzu, Japan) at a wavelength of $480 \mathrm{~nm}$ to determine the concentration of DOX developed in the nanoparticles. After that, the drug loading efficiency in the nanoparticles was calculated with Eq. (1).

Drug Loading Efficiency (DLE)

$$
=\frac{\left(\text { Doxorubicin }_{\text {initial }}\right)-\left(\text { Doxorubicin }_{\text {final }}\right)}{\left(\text { Doxorubicin }_{\text {initial }}\right)} \times 100 \%
$$

Drug loading capacity was determined by the calculation the drug loaded in nanoparticles divided by the amount of nanoparticlesin $\mathrm{mg} / \mathrm{g}$ unit.

\section{In vitro drug release of doxorubicin from MNPs}

Testing of drug release speed was carried out by dispersing $10 \mathrm{mg}$ of MNPs-OA-chitosan (containing DOX) into a dialysis tube (dialysis tubing cellulose membrane, avg flat width $76 \mathrm{~mm}$ ) which had been activated. Next, it was incubated in a container of

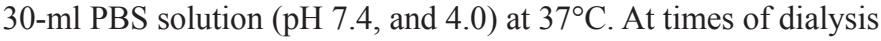
$1,2,4,6,12,24,36,48,60,72$, and 84 hours, $5 \mathrm{ml}$ of supernatant was taken and replaced with fresh PBS. The DOX concentration in the supernatant was determined by a Visible spectrophotometer at $480 \mathrm{~nm}$ wavelength. Pre-determined concentrations of DOX solutions were employed for a standard curve.

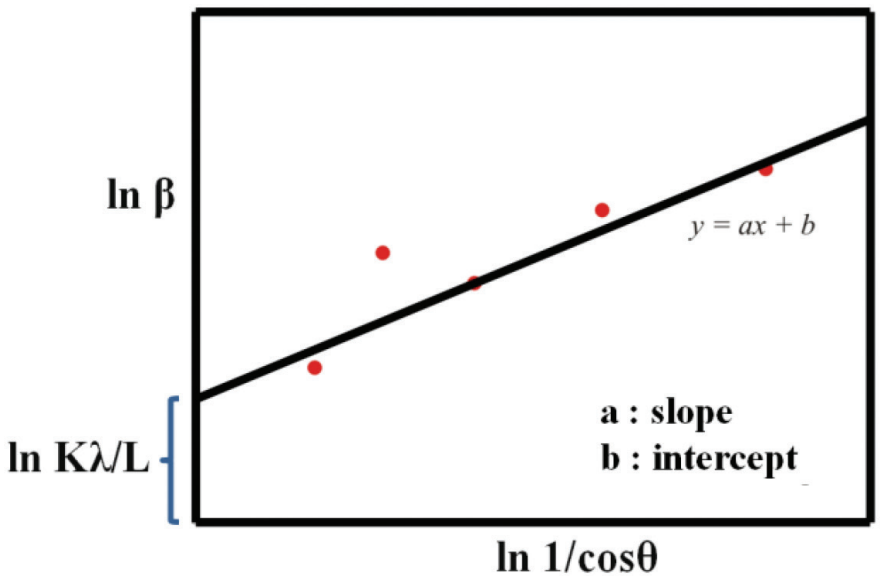

Figure 1. Modified plot sherrer equation.

\section{RESULTS AND DISCUSSION}

Synthesis of MNPs and the effect of surface modification based on oleic acid and TPP/sulfate cross-linked chitosan obtained by ex situ and in situ coprecipitations toward material properties

The purpose of this study was to investigate the effect of synthesis method on properties and magnetic characteristics of the materials. In this study, the coprecipitation method was divided into ex situ and in situ coprecipitations. During the magnetic synthesis process of nanoparticles, there was a change in the color of Fe solution along with the ammonia water addition. These color changes indicated iron oxide phase changes in the stages of nanoparticles' magnetic formation (Ahn et al., 2012).

In ex situ method, it could be described that nanoparticles formed a core-shell structure consisting of MNPs in the core section with OA-chitosan bonded to crosslinked TPP/Sulfate in the shell part as seen in Figure 2. Meanwhile, the in situ method of nanoparticles could be described when MNPs were scattered on the surface of OA-chitosan crosslinked by TPP/sulfate (surface modifier as a matrix carrying MNPs).

SEM images of bare MNPs and modified MNPs nanoparticles synthesized by the ex situ and in situ methods are shown in Figure 3. SEM results showed that nanoparticles (MNPs-OA-chitosan) produced by ex situ method were spherical (Fig. 3b). The shape of the resulted nanoparticles by in situ method (addition of $1 \mathrm{ml}$ oleic acid) resembled a sheet although a small area on its surface looked like spherical shape (Fig. 3c), whereas in lower composition of oleic acid $(0.1 \mathrm{ml})$, it showed porous sheets with a smoother surface (Fig. 3d). These results indicate that nanoparticles (MNPs-OA-chitosan) obtained by ex situ method exhibits more spherical shape than those in situ method.

Nanoparticles produced by both ex situ and in situ coprecipitation methods seemed to form clusters. According to Janke et al. (2014), it is because oleic acid has tendency to form a collection of particles depending on $\mathrm{pH}$ conditions and the protonation state of oleic acid molecules. Theoretically, in the addition of oleic acid, it is expected to produce better nanoparticles with spherical shapes due to the vesicle formation. In the ex situ method, oleic acid was dissolved with ethanol and added to MNPs which had been conditioned at neutral $\mathrm{pH}$ regions (7.0-7.5). Under these conditions, oleic acid would be in the oil phase and

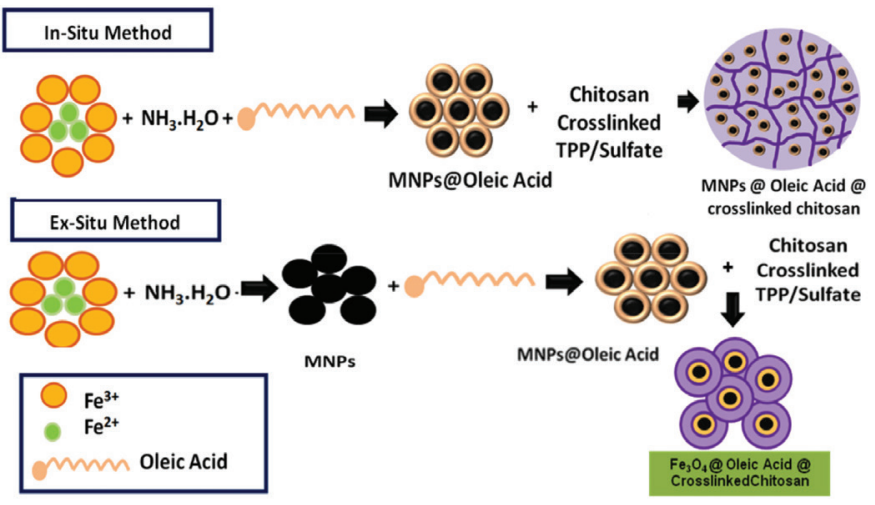

Figure 2. The reaction mechanism which might be occured with the ex-situ and in-situ synthesis processes. 
tended to form the cubosome type vesicles. Meanwhile, in the in situ method, the addition of oleic acid was done in the process of forming MNPs. The solution condition at that time was dominated by bases due to the addition of ammonia water indicated by the solution $\mathrm{pH}>9.2$. During dropwise addition of ammonia, oleic acid can form spherical vesicles which may result in better spherical shape of MNPs when solution $\mathrm{pH}$ reachs 8.5 , however, at higher $\mathrm{pH}$, the spherical vesicle of oleic acid transforms into the tubular/ cylindrical type vesicles (Suga et al., 2016). Since the formation of MNPs by in situ coprecipitation method in this work, occurred at $\mathrm{pH}>9.2$, less spherical MNPs are obtained. Additionally, larger aggregate of MNPs occurred by in situ method because of high water content during the addition of ammonia solution which also
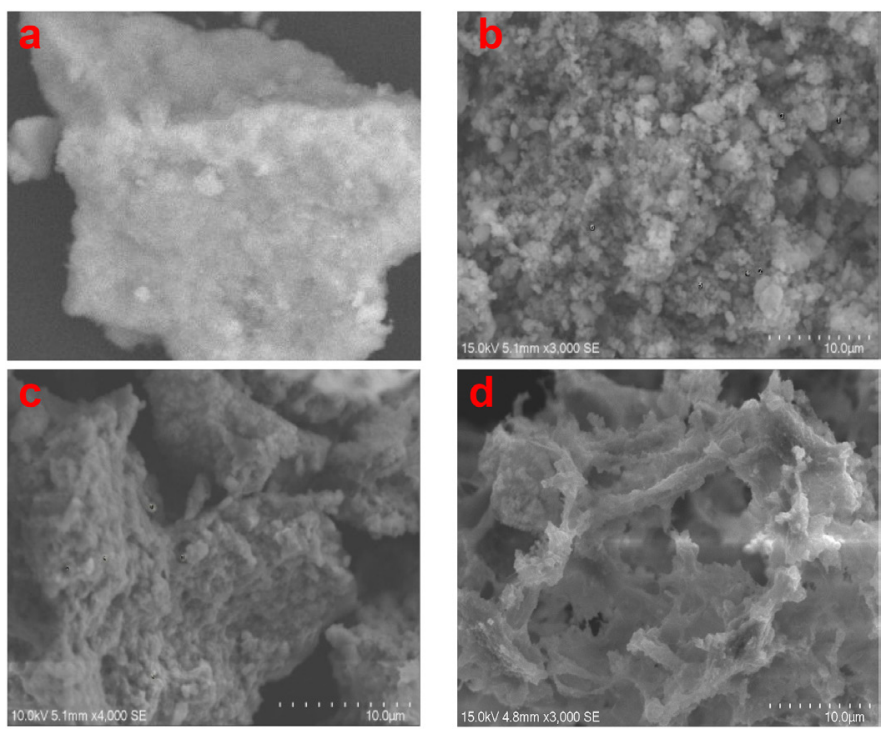

Figure 3. Surface morphology of nanoparticle samples (a) bare MNPs, and MNPs-OA-Chitosan synthesized in (b) Ex-situ coprecipitation (1 ml OA composition), and in-situ coprecipitation with OA compositions (c) $1 \mathrm{ml}$, (d) $0.1 \mathrm{ml}$. contribute to the less spherical MNPs formation. Therefore, it is clear that better spherical shapes of MNPs tended to be obtained in the synthesis method of ex situ compared to in situ coprecipitation as shown by SEM in Figure 3. These results were also confirmed with particle and crystallite sizes of MNPs obtained by in situ method which is larger than ex situ method as shown in Table 1.

The obtained FTIR spectra profile for the sample with ex situ and in situ coprecipitation methods are shown in Figure 4A. There were significant differences in FTIR absorption, especially in the wavenumbers around $\sim 3,500 \mathrm{~cm}^{-1}$ and $1,200-1,350 \mathrm{~cm}^{-1}$ which correlated with oleic acid uptake on the surface of MNPs. The $\mathrm{OH}$ absorption peak at MNPs surface was shown in the area of 3,300-3,400 $\mathrm{cm}^{-1}$. The peak was not seen in the nanoparticle samples produced by in situ coprecipitation method. In the in situ method, FTIR uptake was at an area of $3,550 \mathrm{~cm}^{-1}$ with a weak unwidened absorption profile indicating the presence of free carboxylic acids which did not interact with $\mathrm{OH}$ on the surface of MNPs. In contrast, this absorption peak did not appear in the ex situ method. In addition, there was a shift in absorption from C-O stretching for in situ and ex situ methods in the wavenumber regions of 1,200-1,350 $\mathrm{cm}^{-1}$. The widening of absorption peaks in ex situ method indicated the increasing number of C-O from oleic acid on the surface of MNPs. The symmetrical and asymmetrical $\mathrm{C}-\mathrm{H}$ peaks of oleic acid and chitosan appeared in wavenumbers of 2,950 and $2,880 \mathrm{~cm}^{-1}$. These peaks were not owned by uncoated samples (bare MNPs).

The widening of absorption peaks in ex situ method indicated the increasing number of $\mathrm{C}-\mathrm{O}$ from oleic acid on the surface of MNPs. The symmetrical and asymmetrical C-H peaks of oleic acid and chitosan appeared in wavenumbers of 2,950 and $2,880 \mathrm{~cm}^{-1}$. These peaks were not owned by uncoated samples (bare MNPs). The absorption peak in the $568 \mathrm{~cm}^{-1}$ was from the Fe-O vibration of MNPs. This absorption peak appeared on the three FTIR spectra either for bare MNPs and MNPs-OAchitosan synthesized in ex situ and in situ coprecipitation. Based on the FTIR spectra profile, the interaction between carboxylic acid and MNPs was more dominant in the synthesis using ex situ

Table. 1. Particle size, crystallite size, and iron phase abundance for various nanoparticles composition.

\begin{tabular}{|c|c|c|c|c|}
\hline Parameters & Crystallite size (nm) & Particle size (nm) & $\mathrm{Fe}_{3} \mathrm{O}_{4}(\%)$ & $\mathrm{Fe}_{2} \mathrm{O}_{3}(\%)$ \\
\hline Bare MNPs & 11.90 & $73-162$ & 87.98 & 12.02 \\
\hline \multicolumn{5}{|c|}{ Oleic acid composition (ml) } \\
\hline 1 & 4.73 & $94-149$ & 97.90 & 2.10 \\
\hline 0.5 & 9.93 & $200-333$ & 94.58 & 5.42 \\
\hline 1 & 9.82 & $199-386$ & 85.98 & 14.02 \\
\hline \multicolumn{5}{|c|}{ Ratio of Chitosan: TPP: Sulfate (w/v \%) } \\
\hline $1: 2.5: 7.5$ & 11.21 & $209-331$ & 88.41 & 11.59 \\
\hline \multicolumn{5}{|c|}{ Influence of Coprecipitation Method } \\
\hline Ex-situ & 4.73 & $94-149$ & 97.90 & 2.10 \\
\hline In-situ & 14.64 & $200-461$ & 75.75 & 24.25 \\
\hline
\end{tabular}


coprecipitation method than in situ. The lower curve obtained at the $568 \mathrm{~cm}^{-1}$ indicates that MNPs prepared by ex situ method has higher abundance of $\mathrm{Fe}_{3} \mathrm{O}_{4}$ than that of in situ method.

The MNPs nanoparticle diffraction pattern of non and with chitosan-oleic acid coating produced through in situ and ex situ coprecipitation methods was shown in Figure 4B. All the MNPs samples had the same diffraction peaks at the $2 \theta$ position and were confirmed as the peak of $\mathrm{Fe}_{3} \mathrm{O}_{4}$ with the inverse cubic spinel structure (Silva et al., 2013). This indicated that the coating process has no effect on the crystal structure. However, the peak position of diffractogram $\mathrm{Fe}_{3} \mathrm{O}_{4}$ was known to have similarities with $\gamma-\mathrm{Fe}_{2} \mathrm{O}_{3}$. Therefore, it was necessary to calculate the iron oxide phase abundance in each sample by using MAUD software to determine the percentage of each iron oxide.

From the calculation as presented in Table 1, it was shown that the main component of iron oxide contained in the nanoparticle sample was $\mathrm{Fe}_{3} \mathrm{O}_{4}$. This data were also strengthened by a visual display of black nanoparticles and had an active response to external magnetic fields. The percentage of $\mathrm{Fe}_{3} \mathrm{O}_{4}$ was increased after coating with oleic acid and chitosan synthesized by ex situ coprecipitation method. However, it tends to decrease in nanoparticles synthesized by in situ method. In addition, XRD diffractogram was also used in crystallite size determination with the modified Debye Scherrer equation. There was a correlation between the size of crystallite and the percentage of $\mathrm{Fe}_{3} \mathrm{O}_{4}$. The percentage of $\mathrm{Fe}_{3} \mathrm{O}_{4}$ increased along with smaller crystallite size.

Based on the physical characteristic results of produced nanoparticles, nanoparticles obtained by ex situ coprecipitation method were more suitable as drug delivery agents compared to nanoparticles produced from in situ coprecipitation method. Therefore, further studies related to the effect of material composition in ex situ coprecipitation methods were carried out to determine the optimum composition in producing nanoparticles suitable as a candidate drug delivery agent.

\section{MNPs-OA-chitosan nanoparticles with various oleic acid compositions}

In this study, oleic acid was made in three composition variations. Characterization results with SEM are shown in Figure 5. These results indicated that oleic acid with smaller composition caused particles to form clusters of greater size and non-uniform surface shape/morphology. This was possible since the low composition of oleic acid which interacted with MNPs provided an opportunity for chitosan to also interact with the surface of MNPs. In this study, both of them acted as surface modifiers. As a result, there was a competition between oleic acid and chitosan to interact with MNPs.

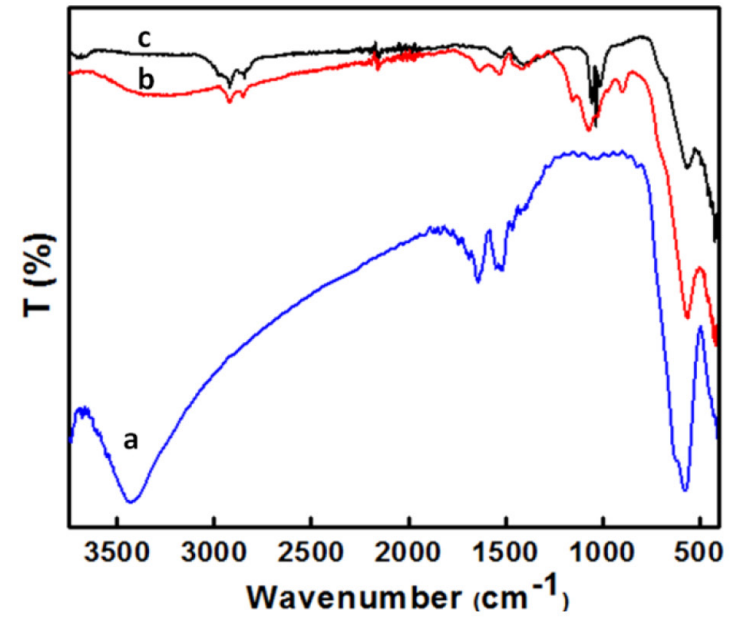

(A)

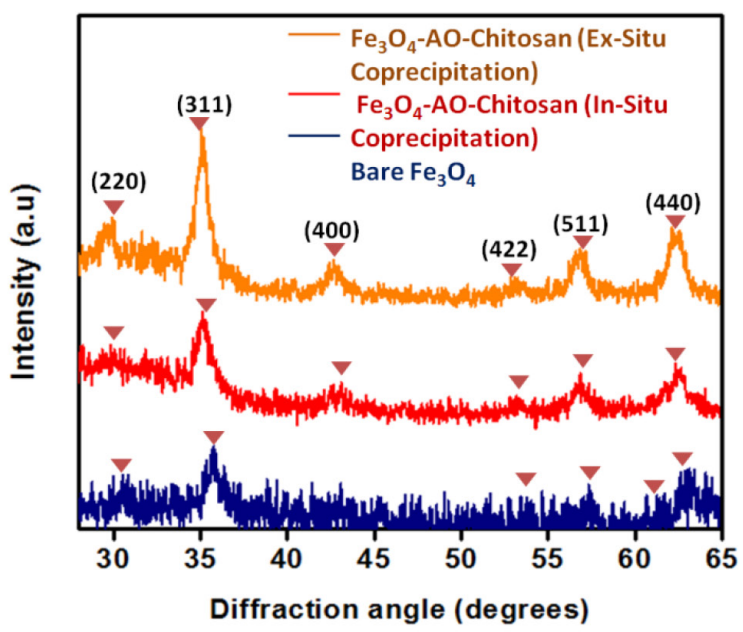

(B)

Figure 4. (A) FTIR spectra in nanoparticle samples (a) bare MNPs, and MNPs-OA Chitosan synthesized by (b) Ex-situ coprecipitation, (c) In-situ coprecipitation. (B) Diffraction pattern on MNPs samples of non and with coating Oleic Acid-Chitosan synthesized by in-situ and ex-situ coprecipitation.
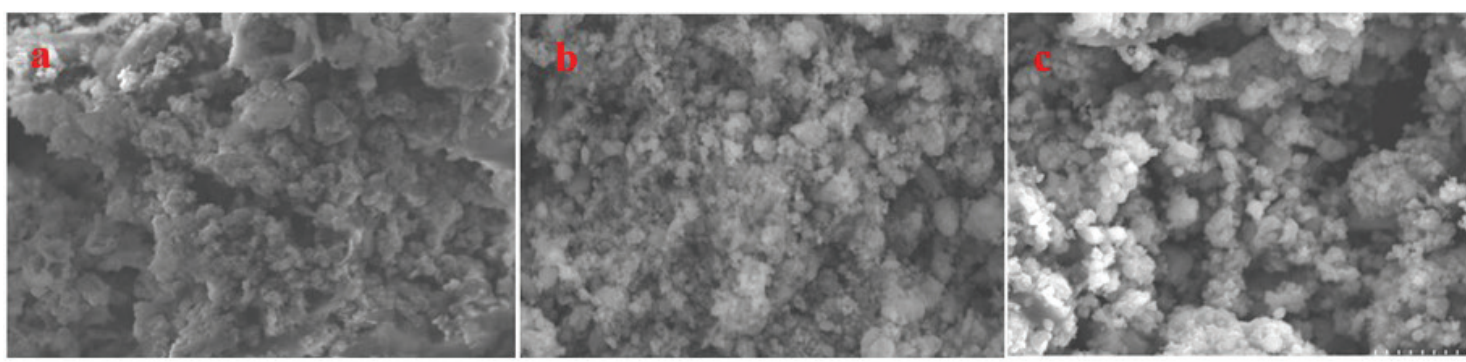

Figure 5. Surface morphology of MNPs-OA-chitosan nanoparticles by adding oleic acid (a) $0.5 \mathrm{ml}$, (b) $1 \mathrm{ml}$, and (c) $1.5 \mathrm{ml}$. 
The different molecular sizes of chitosan and oleic acids caused the cluster formation in a non-uniform size. Nevertheless, in larger composition of oleic acid, the nanoparticle cluster starting to disperse, as a result producing particles that more homogenous in size and shape. It is likely due to the fact that most prominent MNPs interacted with oleic acid and formed a single layer throughout the surface. In the right composition, oleic acid molecules consisting of long and hydrophobic hydrocarbon chains are able to reduce the tensile forces between particles to form clusters. Furthermore, it was able to prevent oxidation of MNPs by forming a protective monolayer on the surface of MNPs. But, at higher composition, oleic acid tends to form bilayers and interact with chitosan to form larger particles (Baharuddin et al., 2018).

Functional group identification of MNPs-OA-chitosan nanoparticles was carried out by FTIR and shown in Figure 6A. Absorption peaks showed the presence of oleic acid on the surface of MNPs were asymmetrical and symmetrical $\mathrm{C}=\mathrm{O}$ peaks at the position of wave numbers of 1,477 and $1,590 \mathrm{~cm}^{-1}$. Oleic acid on the surface of MNPs could form monodentate, bridging, and chelating structures. Based on research results of Jadhav et al. (2013) and Okassa et al. (2007), when the symmetrical and asymmetric uptake differences are more than $100 \mathrm{~cm}^{-1}$, monodentate is more dominant in the MNPs surface.

This monodentate structure is more flexible than bridging and chelating. Furthermore, indication of monodentate structure was shown by the peak that appeared at wavenumber $1,632 \mathrm{~cm}^{-1}$. It was identified as $\mathrm{C}=\mathrm{O}$ asymmetric bond and it was a shift peak from the original peak at $1,710 \mathrm{~cm}^{-1}$ (Lan et al., 2006). In a larger composition, oleic acid could form bilayers on the surface of MNPs. In this condition, it could act as a crosslinker since it can interact with chitosan through the exchange of protons from carboxyl groups $(-\mathrm{COOH})$ in oleic acid with amine groups $\left(-\mathrm{NH}_{2}\right)$ which were free of chitosan. This is consistent with the research of Sailakshmi et al. (2013), which allows the presence of electrons between the amine group and the carboxyl group. The mechanism of possible reaction between oleic acid as a crosslinker in chitosan is shown in Figure 7. The role of oleic acid crosslinker could also affect the nanoparticle size. This caused the produced nanoparticles by adding $1.5 \mathrm{ml}$ of oleic acid to be smaller than by adding $0.5 \mathrm{ml}$ of oleic acid.

XRD diffractogram on MNPs-OA-chitosan nanoparticles samples with different oleic acid compositions was shown in Figure $6 \mathrm{~B}$. This data were then used to determine the size of MNPs and MNPs-OA-chitosan nanoparticles crystallites and calculated through the modified Debye-scherrer equation. The crystallite size of each MNPs-OA-chitosan nanoparticle with different oleic acid compositions was presented in Table 1. Based on the calculation results, the smallest nanoparticle size was obtained with $1 \mathrm{ml}$ oleic acid composition. The addition of oleic acid to more than $1 \mathrm{ml}$ caused the size of the nanoparticle crystallite to increase. Therefore, the optimum composition of oleic acid added as a surface modifier was $1 \mathrm{ml}$.

Magnetic properties of MNPs-OA-chitosan nanoparticles with variations in chitosan composition were known from the characterization results with VSM. MNPs-OAchitosan loop hysteresis with variations in oleic acid composition is shown in Figure 8A. Saturation magnetization obtained in each sample was in range 20.53-32.63 emu/g, respectively, for samples with $0.5-1.5 \mathrm{ml}$ OA coatings. Characterization results with VSM showed that the higher the composition of oleic acid was the higher magnetic value of the saturation would increase. There was a significant difference from the magnetic value of the nanoparticle sample saturation with $\mathrm{Fe}_{3} \mathrm{O}_{4}$ bulk $(62 \mathrm{emu} / \mathrm{g})$. The difference in saturation magnetic values was possible due to the changes from bulk material to nanoparticles. In addition, the presence of non-magnetic layers on the MNPs surface was also a factor in decreasing the magnetic values of nanoparticle saturation compared to $\mathrm{Fe}_{3} \mathrm{O}_{4}$ bulk (Aliakbari et al., 2015). There was a linear correlation between saturation magnetic values with $\mathrm{Fe}_{3} \mathrm{O}_{4}$ content in nanoparticle samples calculated by MAUD software and shown in Figure 8B. The saturation magnetic increase occurred along with the increasing percentage of $\mathrm{Fe}_{3} \mathrm{O}_{4}$ in the

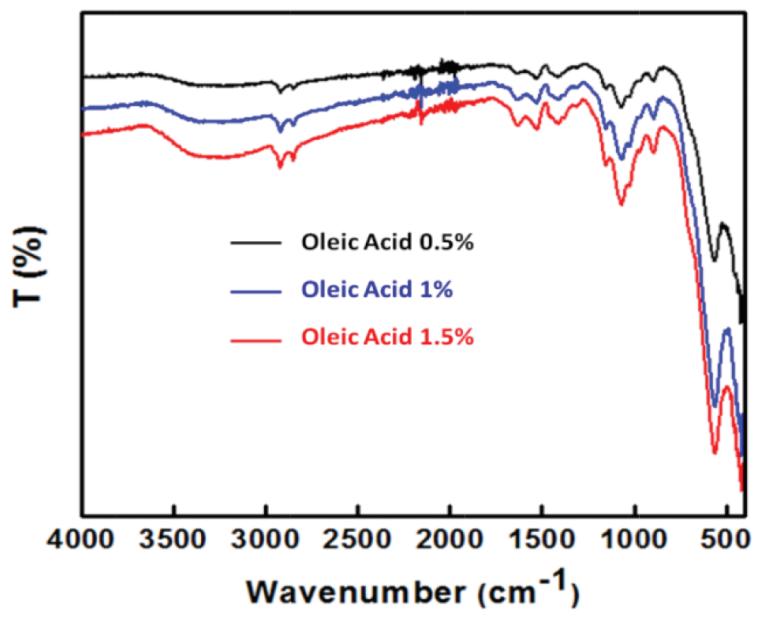

(A)

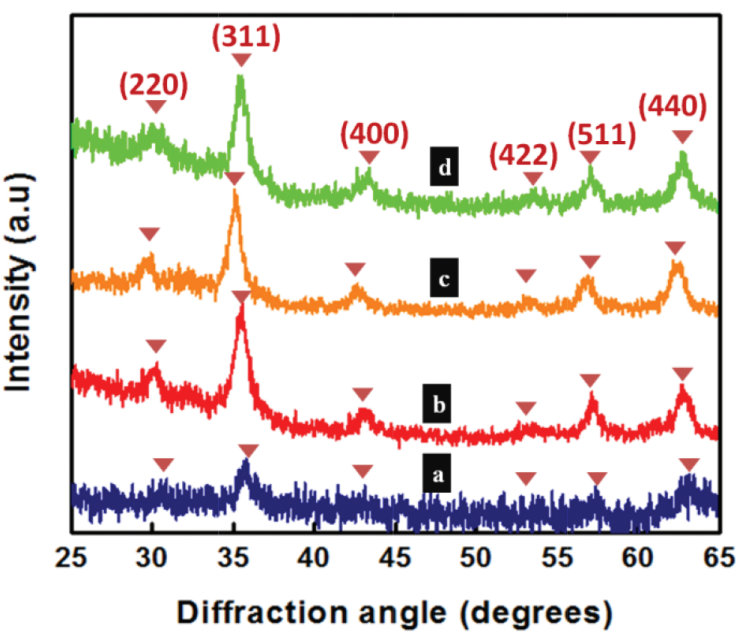

(B)

Figure 6. (A) FTIR spectra in MNPs-OA-chitosan nanoparticles samples with different volume of oleic acid. (B) XRD diffractogram on samples of MNPs-OA-chitosan nanoparticles with variations in oleic acid composition (a) $0 \mathrm{ml}$, (b) $0.5 \mathrm{ml}$, (c) $1 \mathrm{ml}$, and (d) $1.5 \mathrm{ml}$. 
sample. Each sample showed a low coercivity of 6 Oe. As a result, nanoparticles (MNPs-OA-chitosan) could be classified as having superparamagnetic properties. This is consistent with the results of previous studies which states that the superparamagnetics is indicated by a low coercivity value (<10 Oe) (Jadhav et al., 2013).

\section{MNPs-OA-chitosan nanoparticles with different chitosan and crosslinker concentrations}

MNPs-OA-chitosan preparation with $1 \mathrm{ml}$ OA composition and combination of TPP: Sulfate crosslinker 7.5\%:7.5\% with different concentrations of chitosan had been investigated. Increasing the concentration of chitosan resulted in a slightly smaller decrease in particle size and crystallite size. The size of MNPs with and without modifications was shown in Table 1. In contrast, size changes appeared more significant with the addition of TPP/sulfate crosslinking agents which played a role in the nanoparticle formation with more spherical shape. MNPs-OAchitosan preparation with a different combination of TPP:sulfate crosslinkers (OA $1 \mathrm{ml}$ and $1 \%$ chitosan concentration) had also been investigated. The increase in TPP concentration from $2.5 \%$ to $7.5 \%$ had an effect on decreasing the size of crystallites and particles. The smallest size was obtained with TPP concentration of $7.5 \%$. In the aqueous form, chitosan structures can experience conformational widening and become more flexible. This happened due to the repulsion of the electrostatic charge produced by the chitosan polymer (Lee et al., 2013; Obaidat et al., 2010).

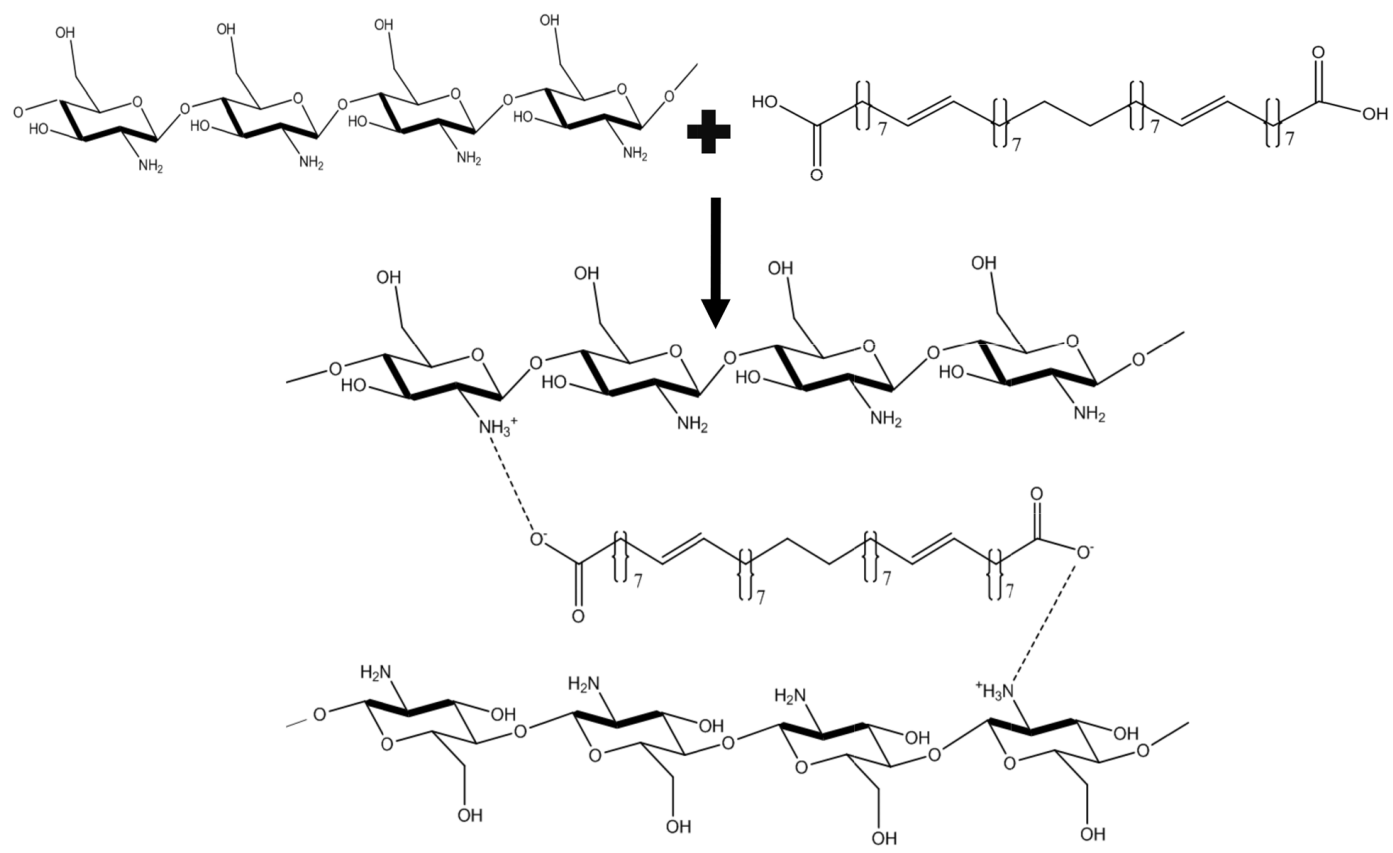

Figure 7. Ionic interaction between oleic acid and chitosan by proton exchange

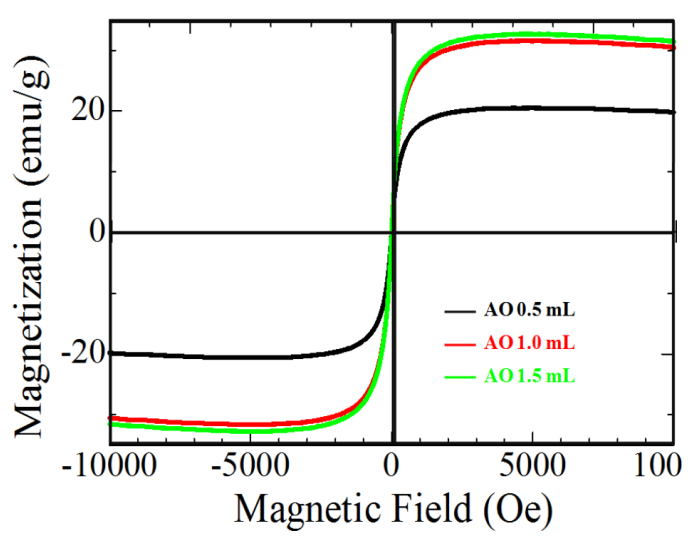

(A)

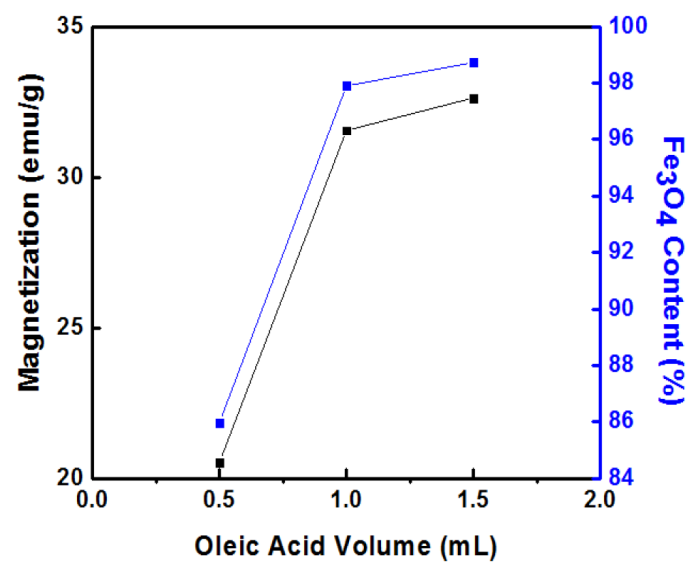

(B)

Figure 8. (A) Hysteresis loop on MNPs-OA-chitosan samples with the addition of OA. (B) Correlation between oleic acid volume, magnetic saturation, and $\mathrm{Fe}_{3} \mathrm{O}_{4}$ percentage in nanoparticle samples with different $\mathrm{OA}$ compositions 
The flexible structure of chitosan strongly supported the spherical shape formation of nanoparticles when chitosan interacted intramolecularly with TPP and sulfate crosslinkers. These results are consistent with the research of Shu and Zhu (2002) which states that the combination of TPP and sulfate plays a role in the formation of spherical morphology of nanoparticles. In addition, the interaction of chitosan with TPP/Sulfate also affects the size of nanoparticles. Research results by Jonassen et al. (2012) also supports data for our study. The high ratio of TPP concentration to chitosan could reduce the occurrence of larger particle formation if the ionic gelation process occured in a short time (no more than 24
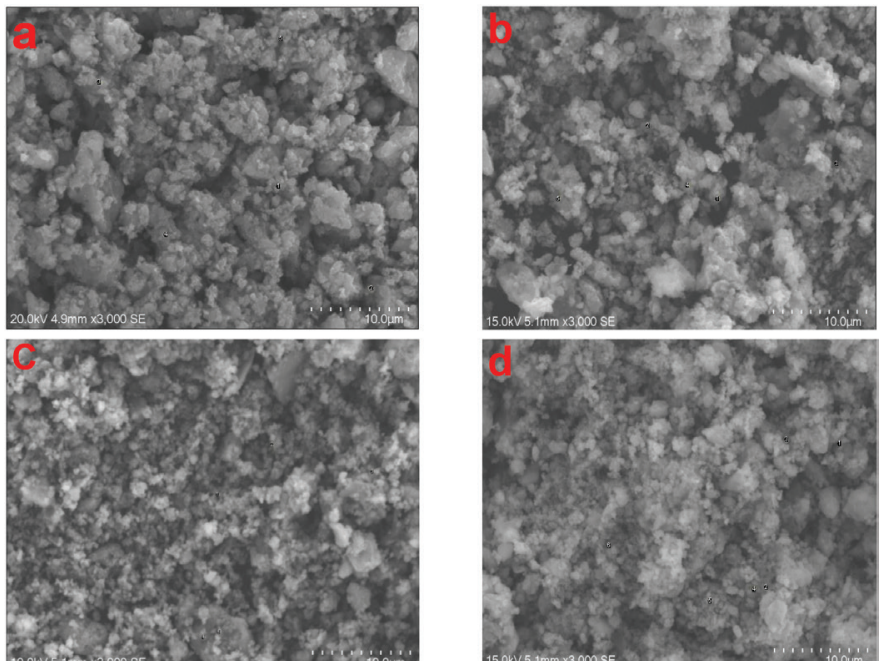

Figure 9. Surface morphology of MNPs-OA-chitosan nanoparticles with (a) $0.5 \%$ chitosan concentration, $1 \mathrm{ml}$ composition of OA, and TPP: sulfate concentration ratio (7.5\%: 7.5\%), and nanoparticle morphology with $1 \%$ chitosan concentration, OA composition of $1 \mathrm{ml}$, TPP: Sulfate ratio of (b) $2.5 \%$ : $7.5 \%$, (c) $5 \%: 7.5 \%$, and (d) $7.5 \%: 7.5 \%$ each with $3,000 \times$ magnification. hours). Surface morphology of MNPs-OA-chitosan nanoparticles with different chitosan concentrations and different TPP:sulfate concentration ratios were shown in Figure 9.

FTIR spectra in MNPs-OA-chitosan samples with different concentrations of chitosan, different TPP:sulfate was shown in Figure 10A and B. The presence of chitosan was shown by absorption in the $1,317 \mathrm{~cm}^{-1}$ wave area corresponding to $\mathrm{C}-\mathrm{N}$ vibration. The uptake was only found in samples of MNPs which were modified on the surface. Doublet uptake in the wave region $2,880-2,950 \mathrm{~cm}^{-1}$ was also a symmetrical and asymmetrical C-H absorption of chitosan.

Whereas, the presence of TPP functional groups was shown by the absorption of P-O-P stretching which appeared in the fingerprint area, at wave $892 \mathrm{~cm}^{-1}$. The intensity of uptake showing the presence of chitosan decreased with increasing TPP:sulfate concentration ratio to form nanoparticles. This indicated the existence of electrostatic interactions between TPP/ sulfate and chitosan (Martins et al., 2012; Mwangi et al., 2016). In general, tripolyphosphoric groups from TPP interact with amine groups from chitosan in ionic bonds. This interaction triggers the ionic gelation process which causes the conversion of polymer chitosan to form nanoparticles. The interaction between chitosanTPP is estimated to be more dominant than chitosan oleic acid. This is supported by SEM images which show more spherical morphology of nanoparticles.

Diffractogram of XRD of MNPs and MNPs-OAChitosan nanoparticles with different concentrations of chitosan and crosslinking agents were shown in Figure 11A and B. There was no change in crystal structure as evidenced by the diffraction peaks at the same $2 \theta$ position for all the diffractograms. However, there was a change in crystallite size and lattice parameters. There was a correlation between TPP concentration and changes in lattice parameters. The lattice parameters reflected the bond distance between $\mathrm{Fe}$ and $\mathrm{O}$ atoms in the structure of MNPs. The

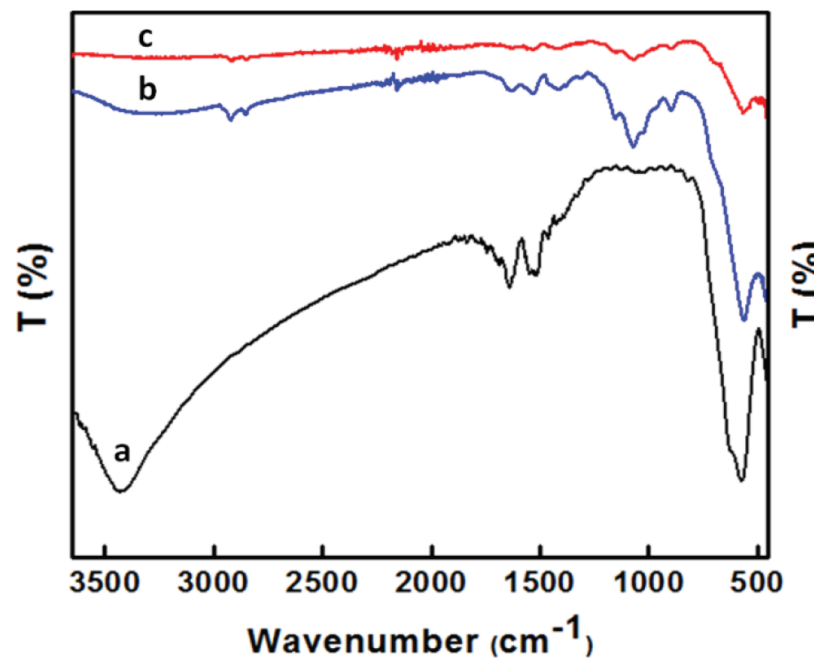

(A)

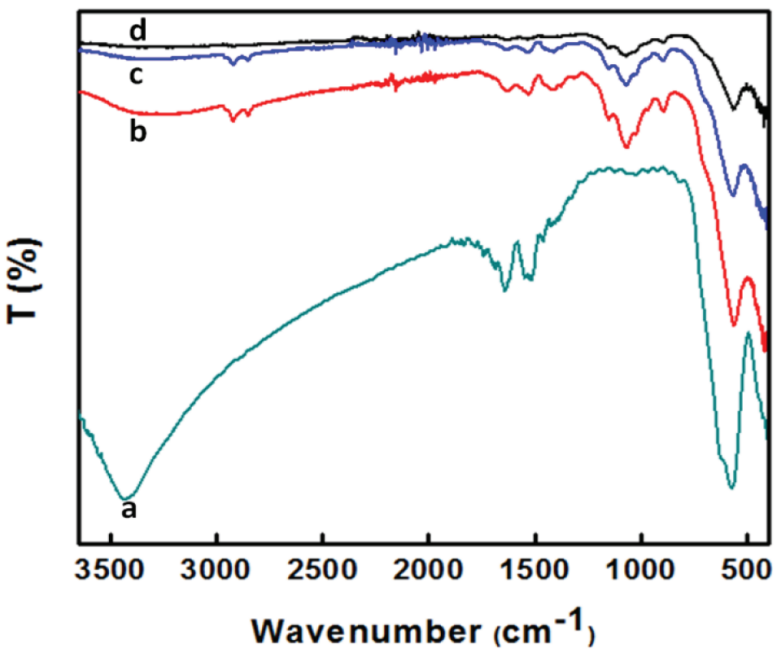

(B)

Figure 10. (A) Nanoparticle FTIR spectra (a) bare MNPs, and MNPs-OA-chitosan nanoparticles with composition of 0.5 ml OA, TPP: Sulfate 7.5\%: 7.5\% and chitosan concentration (b) $1 \%$, (c) $0.5 \%$, ((B) Nanoparticle FTIR spectra (a) bare MNPs, and MNPs-OA-Chitosan nanoparticles with $1 \mathrm{ml}$ of OA composition and concentration ratio of Chitosan: TPP: Sulfate of (b) 1: 7.5: 7.5\%, (c) 1: 5: 7.5\%, and (d) 1: 2.5: $7.5 \%$. 


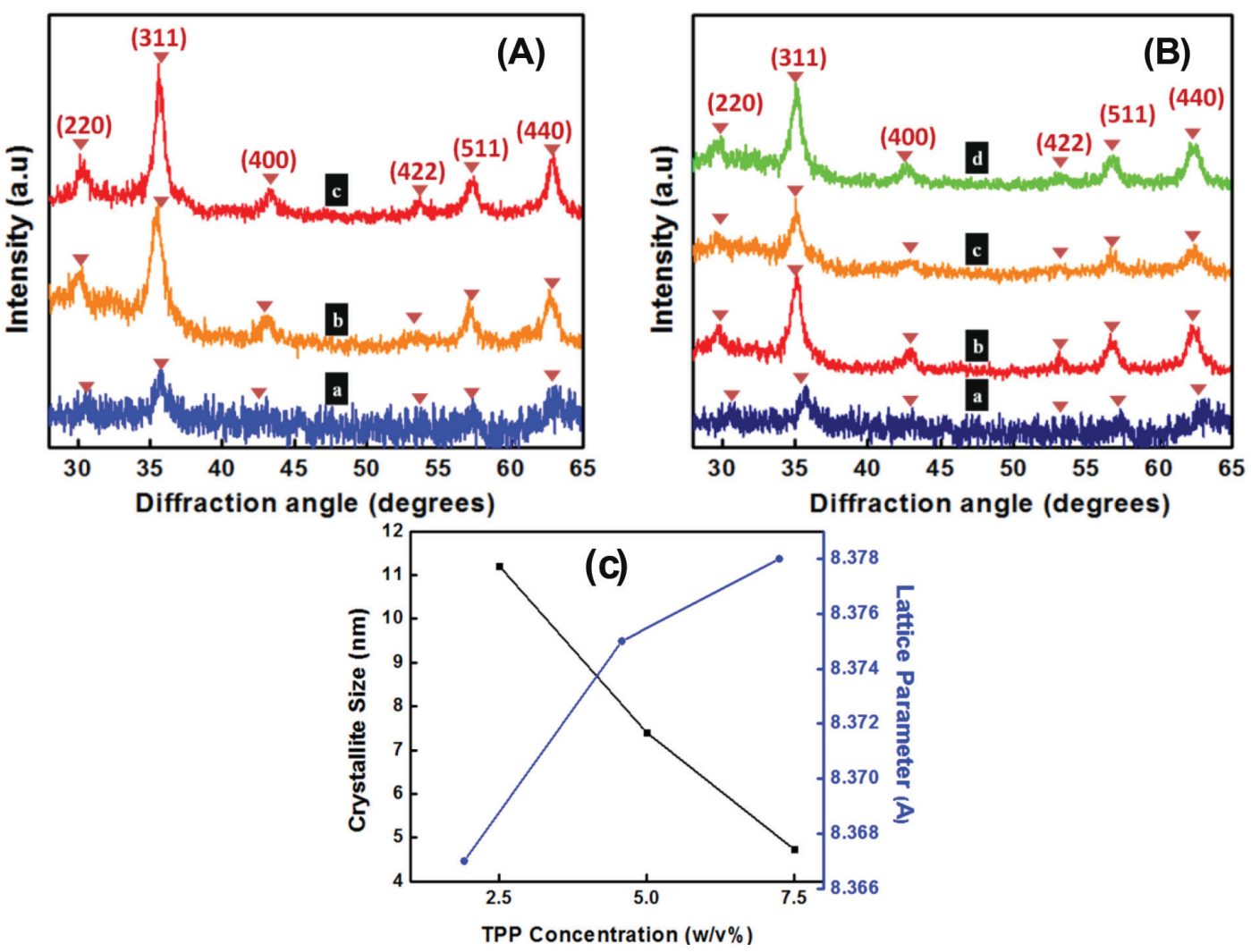

Figure 11. (A) Nanoparticle diffraction pattern (a) bare MNPs, and MNPs-OA-chitosan nanoparticles with chitosan concentration (b) $0.5 \%$, (c) $1 \%$, (B) nanoparticle diffraction pattern (a) bare MNPs, and MNPs-OA-Chitosan nanoparticles with the ratio of Chitosan: TPP: Sulfate (b) 1: 7.5: 7.5\%, (c) 1: 5: 7.5\%, and (d) 1: 2.5: 7.5\%. (C) Correlation graph among TPP concentration, crystallite size and lattice parameters.

correlation can be seen in Figure 11C. Based on JCPDS File19629 for $\mathrm{Fe}_{3} \mathrm{O}_{4}$ and JCPDS File 39-1346 for $\gamma-\mathrm{Fe}_{2} \mathrm{O}_{3}$, the lattice parameters were 8.396 and $8.346 \AA$, respectively. The XRD results showed that the produced MNPs-OA-chitosan samples were between $\mathrm{Fe}_{3} \mathrm{O}_{4}$ and $\gamma-\mathrm{Fe}_{2} \mathrm{O}_{3}$. In addition, Cornell and Schwertmann (2006) mentioned the same thing in terms of lattice parameters for $\mathrm{Fe}_{3} \mathrm{O}_{4}$ (Xu et al., 2013).

\section{Drug loading and in vitro drug release from nanoparticle with the doxorubicin as drug model}

Nanoparticles which had been modified with oleic acid and chitosan surface were then tested for drug loading efficiency using the doxorubicin as a drug model. The doxorubicin drug model was dissolved in phosphate buffer $\mathrm{pH} 7.4$ (DOX concentration was $25 \mathrm{mg} \mathrm{l}^{-1}$ ). Then, $10 \mathrm{mg}$ of nanoparticles were added to the doxorubicin drug solution and stirred for 1 hour to reach equilibrium. Determination of drug loading efficiency was done by measuring the absorbance of doxorubicin solution before and after adding nanoparticles. The drug loading efficiency of DOX development was presented in Table 2. The results showed that nanoparticles with lower chitosan composition resulted in higher drug loading efficiency. This proved that DOX did not interact with chitosan. Doxorubicin basically tends to interact ionically with carboxylic acid groups (from oleic acid). This ionic interaction could occur due to the neutral $\mathrm{pH}$ conditions, carboxylic acids deprotonated to form carboxylic ions which were negatively charged (Xu et al., 2013). Meanwhile, Doxorubicin had protonation to form ammonium ions which were positively charged. But, sometimes in a more complex structure, drug development was not only affected by ionic interactions. The effect of hydrophobicity of MNPs might be existed and it was affecting the drug loading efficiency. The highest loading efficiency of DOX development on MNPs-OAchitosan was $45.45 \%$. The $\mathrm{Fe}_{3} \mathrm{O}_{4}$-loaded Doxorubicin is not examined due to the instability of uncoated $\mathrm{Fe}_{3} \mathrm{O}_{4}$.

The release of drug was determined from MNPs-OAChitosan with the lowest chitosan concentration by dialysis in PBS at different $\mathrm{pH}$. Drug release profile was depicted in Figure 12. The drug release was remarkably affected by the $\mathrm{pH}$ medium. The release rate of Doxorubicin from MNPs-OA-Chitosan increases along with the decrease of $\mathrm{pH}$. It could be attributed to the instability of chitosan in acid condition ( $\left.\mathrm{p} K_{\mathrm{a}}=6.5-6.8\right)$ (Patil et al., 2016). Moreover, the carboxylic acid group of oleic acid $\left(\mathrm{p} K_{\mathrm{a}}=4.8\right)$ (Janke et al., 2014) should be protonated at lower $\mathrm{pH}$. The weakening interaction between DOX and carboxylic group of oleic acid result in drug release from nanoparticle. This result may be explained by the fact that loaded DOX was driven by combination of ionic bonding and hydrophobic effect. At $\mathrm{pH}$ 4.0, which mimics endosomal conditions, the cummulative release of DOX from MNPs-OA-Chitosan reached $70.83 \%$ within 84 hours. 
Table 2. Drug loading efficiency of DOX for various nanoparticles composition.

\begin{tabular}{cccc}
\hline Chitosan (wt \%) & Oleic acid (ml) & $\begin{array}{c}\text { Drug loading } \\
\text { effiency (\%) }\end{array}$ & $\begin{array}{c}\text { Loading capacity } \\
\text { (mg Dox/g NPs) }\end{array}$ \\
\hline 0.5 & 1 & 45.45 & 35.29 \\
1 & 1 & 34.10 & 26.47 \\
2 & 1 & 20.45 & 15.88 \\
1 & 1 & 32.65 & 25.26 \\
1 & 1.5 & 34.78 & 28.23 \\
\hline
\end{tabular}

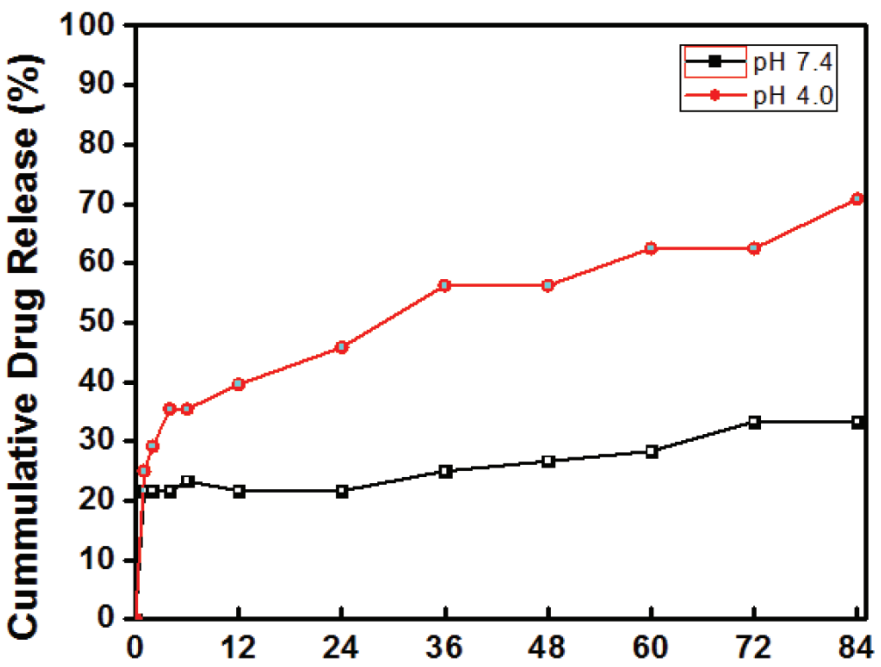

Figure 12. Doxorubicin (DOX) release from MNPs-OA-Chitosan in (a) simulated physiological pH 7.4 and (b) simulated lysosome/endosome $\mathrm{pH} 4.0$.

However, only $33.33 \%$ of DOX could be released at physiological condition ( $\mathrm{pH}$ 7.4). Such results indicated that MNPs-OA-Chitosan has $\mathrm{pH}$ sensitive properties which can respond to the acidic $\mathrm{pH}$ in cancer intracellular organelles by releasing higher amount of DOX in comparison to the physiological $\mathrm{pH}$.

\section{CONCLUSION}

MNPs with surface modifications of oleic acid and chitosan crosslinked by sulfate and TPP have been prepared through the synthesis method of ex situ and in situ coprecipitations. Surface morphology, particle size, and crystallite size of nanoparticles have been investigated. Spherical shapes and smaller sizes tend to be obtained by ex situ coprecipitation method. There is a linear correlation between the percentage of iron oxide $\left(\mathrm{Fe}_{3} \mathrm{O}_{4}\right)$ and the magnetic properties of material, where the higher the percentage of $\mathrm{Fe}_{3} \mathrm{O}_{4}$ is, the greater saturation magnetic value will be. Nanoparticles can also be classified as superparamagnetic because they have low coercivity. The efficiency of drug loading from MNPs-OA-chitosan (up to $45 \%$ ) can occur due to ionic interactions and the hydrophobic effects between DOX-with MNPs-OA-chitosan. The drug release profile shows that at lower $\mathrm{pH}$ conditions (following the lysosome/endosome $\mathrm{pH}$ ) drug release takes place faster than in neutral $\mathrm{pH}$ (physiological $\mathrm{pH}$ ). Cummulative drug release reaches $70.83 \%$ at 84 hours in PBS pH 4.0. Based on the characterization data which includes size, shape, percentage of $\mathrm{Fe}_{3} \mathrm{O}_{4}$, and profile of drug release and drug loading, it can be concluded that MNPs-OA-chitosan synthesized by ex situ coprecipitation method can be used as candidates for drug delivery agents.

\section{ACKNOWLEDGMENTS}

All authors would like to thank Brawijaya University and Gifu University (Prof. Lim Lee Wah) for providing research facilities. This research was partially supported by Ministry of Research, Technology, and Higher Education of The Republic of Indonesia through the research scheme of World Class Research 2019 .

\section{CONFLICT OF INTEREST}

All authors confirmed that there is no conflict of interest.

\section{REFERENCES}

Ahn T, Kim JH, Yang HM, Lee JW, Kim JD. Formation pathways of magnetite nanoparticles by coprecipitation method. J Phys Chem C, 2012; 116:6069-76.

Aliakbari A, Seifi M, Mirzaee S, Hekmatara H. Influence of different synthesis conditions on properties of oleic acid-coated- $\mathrm{Fe}_{3} \mathrm{O}_{4}$ nanoparticles. Mater Sci Poland, 2015; 33:100-6.

Baharuddin AA, Ang BC, Hussein NAA, Andriyana A, Wong YH. Mechanisms of highly stabilized ex-situ oleic acid-modified iron oxide nanoparticles functionalized with 4-pentynoic acid. Mat Chem Phys, 2018; 203:212-22.

Banerjee A, Qi J, Gogoi R, Wong J, Mitragotri S. Role of nanoparticle size, shape and surface chemistry in oral drug delivery. $\mathrm{J}$ Control Release, 2016; 238:176-85.

Bloemen M, Brullot W, Luong TT, Geukens N, Gils A, Verbiest T. Improved functionalization of oleic acid-coated iron oxide nanoparticles for biomedical applications. J Nanoparticle Res, 2012; 14:1-10.

Cho K, Wang X, Nie S, Chen Z, Shin DM. Therapeutic nanoparticles for drug delivery in cancer. Clin Cancer Res, 2008; 14: $1310-6$.

Cornell RM, Schwertmann U. The iron oxides: structure, properties, reactions, occurrences and uses. 2nd edition, Wiley-VCH GmbH, Weinheim, New York, 2006.

De Jong WH, Borm PJ. Drug delivery and nanoparticles: hazards and application. Int J Nanomedi, 2008; 3:133-49.

Dumitriu S, Dumitriu M. . Hydrogels as support for drug delivery systems. In Dumitriu S (ed.). Polyaccharides in medicinal application. Marcel Dekker Inc, New York, NY, pp. 631-49, 1996.

Jadhav NV, Prasad AI, Kumar A, Mishra R, Dhara S, Babu KR, Prajapat CL, Misra NL, Ningthoujamb RS, Pandey BN, Vatsa RK. Synthesis of oleic acid functionalized $\mathrm{Fe}_{3} \mathrm{O}_{4}$ magnetic nanoparticles and studying their interaction with tumor cells for potential hyperthermia applications. Colloids Surf B Biointerfaces, 2013; 108:158-68.

Jain KK. Drug delivery systems - an overview. In Jain KK (ed.). Drug delivery systems. Humana Press, Switzerland, 2008.

Janke JJ, Bennett WFD, Tielemen DP. Oleic acid phase behavior from molecular dynamics simulations. Langmuir, 2014; 30:10661-7.

Jonassen H, Kjoniksen AL, Hiorth M. Effects of ionic strength on the size and compactness of chitosan nanoparticles. Colloid Polym Sci, 2012; 290:919-29.

Lan Q, Liu C, Yang F, Liu S, Xu J, Sun D. Synthesis of bilayer oleic acid-coated $\mathrm{Fe}_{3} \mathrm{O}_{4}$ nanoparticles and their application in $\mathrm{pH}$-responsive pickering emulsions. J Colloid Interface Sci, 2006; 310:260-9.

Lee DW, Lim C, Israelachvili JN, Hwang DS. Strong adhesion and cohesion of chitosan in aqueous solutions. Langmuir, 2013; 29:14222-9.

Majeed MI, Lu Q, Yan W, Li Z, Hussain I, Tahir MN, Tremel W, Tan B. Highly water-soluble magnetic iron oxicde $\left(\mathrm{Fe}_{3} \mathrm{O}_{4}\right)$ nanoparticles for drug delivery: enhanced in vitro therapeutic efficacy of doxorubicin and MION conjugates. J Mater Chem B, 2013; 1:2874-84. 
Martins AF, De Oliviera DM, Pereira AGB, Rubira AF, Muniz EC. Chitosan/TPP microparticles obtained by microemulsion method applied in controlled release of heparin. Int J Biol Macromol, 2012; 51:1127-33.

Monshi A, Foroughi MR, Monshi MR. Modified scherrer equation to estimate more accurately nano-crystallite size using XRD. WJNSE, 2012; 2:154-60.

Mwangi WW, Ho KW, Ooi CW, Tey BT, Chan ES. Facile method for forming ionically cross-linked chitosan microcapsules from pickering emulsion templates. Food Hydrocoll, 2016; 55:26-33.

Obaidat R, Al-Jbour N, Al-Sou'd K, Sweidan K, Al-Remawi M, Badwan A. Some physico-chemical properties of low molecular weight chitosans and their relationship to conformation in aqueous solution. $\mathrm{J}$ Solution Chem, 2010; 39:575-88.

Okassa LN, Marchais H, Douziech-Eyrolles L, Herve K, Cohen-Jonathan S, Munnier E, Souce M, Linassier C, Dubois P, Chourpa I. Optimization of iron oxide nanoparticles encapsulation within poly (D,LLactide-Co-Glycolide) sub-micron particles. Eur J Pharm Biopharm, 2007; 67:31-8.

Patil S, Bhatt P, Lalani R, Amrutiya J, Vhora I, Kolte A, Misra A. Low molecular weight chitosan-protamine conjugate for siRNA delivery with enhanced stability and transfection efficiency. RSC Adv, 2016; 6:110951-63.

Pereira da Silva S, Costa de Moraes D, Samios D. Iron oxide nanoparticles coated with polymer derived from epoxidized oleic acid and Cis-1 , 2-cyclohexanedicarboxylic anhydride: synthesis and characterization. J Mater Sci Eng, 2016; 5:1-7.

Sailakshmi G, Mitra T, Gnanamani A. Engineering of chitosan and collagen macromolecules using sebacic acid for clinical applications. Prog Biomater, 2013; 2:1-11.

Suga K, Kondo D, Otsuka Y, Okamoto Y, Umakoshi, H. Characterization of aqueous oleic acid/oleate dispersions by fluorescent probes and raman spectroscopy. Langmuir, 2016; 32(30):7606-12.

Shu XZ, Zhu KJ. Controlled drug release properties of ionically cross-linked chitosan beads: the influence of anion structure. Int J Pharm, 2002; 233:217-25.

Silva VAJ, Andrade PL, Silva MPC, Bustamante AD, Valladares LDS, Aguiar JA. Synthesis and characterization of $\mathrm{Fe}_{3} \mathrm{O}_{4}$ nanoparticles coated with fucan polysaccharides. IJ Magn Magn Mater, 2013; 343:138-43.

Singh KS, Vasundhara M. Optimization of formulation parameters for controlled drug delivery from metformin hydrochloride loaded chitosan/TPP microspheres. Pharma Innovation, 2015; 4:38-42.
Snipstad S, Westorm S, Morch Y, Afadzi M, Aslund Andreas KO, Davies CL. Contact-mediated intracellular delivery of hydrophobic drugs from polymeric nanoparticles. Cancer Nanotechnol, 2014; 5:1-18.

Tran TTD, Vo TV, Tran PHL. Design of iron oxide nanoparticles decorated oleic acid and bovine serum albumin for drug delivery. Chem Eng Res Des, 2015; 94:112-8.

Ulbrich W, Lamprecht A. Targeted drug-delivery approaches by nanoparticulate carriers in the therapy of inflammatory diseases. J R Soc Interface, 2010; 7:S55-6.

Velusamy P, Hung SC, Shritama A, Kumar GV, Jeyanthi V, Pandian K. Synthesis of oleic acid coated iron oxide nanoparticles and its role in anti-biofilm activity against clinical isolates of bacterial pathogens. $\mathrm{J}$ Taiwan Inst Chem Eng, 2016; 59:450-6.

Voicu G, Geanaliu-Nicolae RE, Pirvan AA, Andronescu E, Iordache F. Synthesis, characterization and bioevaluation of drug-collagen hybrid materials for biomedical applications. Int J Pharm, 2016; 510 474-84.

Xu Z, Guo M, Yan H, Liu K. Enhanced loading of doxorubicin into polymeric micelles by a combination of ionic bonding and hydrophobic effect, and the $\mathrm{pH}$-sensitive and ligand-mediated delivery of loaded drug. Reactive Funct Polym, 2013; 73:564-72.

How to cite this article:

Wulandari IO, Sulistyarti H, Safitri A, Santjojo DJDH, Sabarudin A. Development of synthesis method of magnetic nanoparticles modified by oleic acid and chitosan as a candidate for drug delivery agent. J Appl Pharm Sci, 2019; 9(07):001-011. 